Bull. Korean Math. Soc. 51 (2014), No. 4, pp. 1175-1186

http://dx.doi.org/10.4134/BKMS.2014.51.4.1175

\title{
SELF-DUAL CODES AND FIXED-POINT-FREE PERMUTATIONS OF ORDER 2
}

\author{
HYUn Jin KIM
}

\begin{abstract}
We construct new binary optimal self-dual codes of length 50. We develop a construction method for binary self-dual codes with a fixed-point-free automorphism of order 2 . Using this method, we find new binary optimal self-dual codes of length 52. From these codes, we obtain Lee-optimal self-dual codes over the $\operatorname{ring} \mathbb{F}_{2}+u \mathbb{F}_{2}$ of lengths 25 and 26.
\end{abstract}

\section{Introduction}

We have been interested in finding binary optimal self-dual codes. Many binary optimal self-dual codes of length 50 are obtained in $[3,4,7,10,20]$. We find binary optimal self-dual codes of length 52 in $[3,4,10,12,21,29,31$, 30, 32]. Also, codes over the ring $\mathbb{F}_{2}+u \mathbb{F}_{2}$ have been also interesting due to their usefulness for construction of Hermitian modular forms [1] and Gaussian lattices [9]. We find all self-dual codes over $\mathbb{F}_{2}+u \mathbb{F}_{2}$ of length up to 8 in [9]. All Lee-extremal and Lee-optimal self-dual codes over $\mathbb{F}_{2}+u \mathbb{F}_{2}$ of lengths 9 through 24 with a nontrivial automorphism of odd order are classified in $[18,19,22,24,25]$.

If $\mathcal{C}$ is a self-dual code over the ring $\mathbb{F}_{2}+u \mathbb{F}_{2}$ of length $n$, then the Gray image of $\mathcal{C}$ is a binary self-dual code of length $2 n$ with a fixed-point-free automorphism of order $2[9]$.

In this paper, we develop a construction method for a self-dual code of length $2 n+2$ which has a fixed-point-free automorphism of order 2 from a self-dual code of length $2 n$ with a fixed-point-free automorphism of order 2 . Using this extension method, we find 184 new inequivalent optimal self-dual $[52,26,10]$ codes with a fixed-point-free automorphism of order 2 from two new optimal self-dual $[50,25,10]$ codes which we construct using the method in [5]. From these codes, we also obtain Lee-optimal self-dual codes of lengths 25 and 26

Received October 6, 2013; Revised January 30, 2014.

2010 Mathematics Subject Classification. Primary 94B05; Secondary 11T71.

Key words and phrases. automorphism, extremal code, optimal code, self-dual code.

The author was supported by Basic Science Research Program through the National Research Foundation of Korea(NRF) funded by the Ministry of Education(2009-0093827). 
over the ring $\mathbb{F}_{2}+u \mathbb{F}_{2}$. Computations for the results are carried out using MAGMA.

The rest of the paper is organized as follows. In Section 2, we construct new binary optimal self-dual codes of length 50 and present a construction method for binary self-dual codes with a fixed-point-free automorphism of order 2 . Using this method, we construct new binary optimal self-dual codes of length 52 from binary optimal self-dual codes of length 50. In Section 3, we construct Lee-optimal self-dual codes over $\mathbb{F}_{2}+u \mathbb{F}_{2}$ of lengths 25 and 26 from the results in Section 3.

\section{Binary codes}

An $[n, k]$ linear code $\mathcal{C}$ over the binary field $\mathbb{F}_{2}$ is a $k$-dimensional subspace of $\mathbb{F}_{2}^{n}$. The Hamming weight of a vector in $\mathbb{F}_{2}^{n}$ is the number of its nonzero coordinates. The minimum weight $d$ of a code $\mathcal{C}$ is the smallest weight of its nonzero codewords and $\mathcal{C}$ is called an $[n, k, d]$ code. For every $\mathbf{u}=\left(u_{1}, u_{2}, \ldots, u_{n}\right)$ and $\mathbf{v}=\left(v_{1}, v_{2}, \ldots, v_{n}\right)$ from $\mathbb{F}_{2}^{n}, \mathbf{u} \cdot \mathbf{v}=u_{1} \cdot v_{1}+u_{2} \cdot v_{2}+\cdots+u_{n} \cdot v_{n}$ defines the Euclidean inner product in $\mathbb{F}_{2}^{n}$. The dual code of $\mathcal{C}$ is $\mathcal{C}^{\perp}=\left\{\mathbf{u} \in \mathbb{F}_{2}^{n} \mid \mathbf{u} \cdot \mathbf{v}=\right.$ 0 for all $\mathbf{v} \in \mathcal{C}\}$, and $\mathcal{C}^{\perp}$ is a linear $[n, n-k]$ code. If $\mathcal{C} \subseteq \mathcal{C}^{\perp}$, then $\mathcal{C}$ is called self-orthogonal, and if $\mathcal{C}=\mathcal{C}^{\perp}$, then we call it self-dual. A self-dual code with all codewords of weight divisible by 4 is called doubly-even and a self-dual code with some codeword of weight not divisible by 4 is called singly-even. Upper bounds on the minimum weight of these codes are given by [27, 28].

Theorem 2.1. Let $\mathcal{C}$ be an $[n, n / 2, d]$ self-dual binary code. If $\mathcal{C}$ is singly-even, then

(i) when $n \not \equiv 22(\bmod 24), d \leq 4\lfloor n / 24\rfloor+4$,

(ii) when $n \equiv 22(\bmod 24), d \leq 4\lfloor n / 24\rfloor+6$.

If $\mathcal{C}$ is doubly-even, then $d \leq 4\lfloor n / 24\rfloor+4$.

The self-dual codes meeting the bounds of Theorem 2.1 are called extremal, and the self-dual codes with the largest minimum weight for a given length are called optimal [17]. Two binary codes are equivalent if one can be obtained from the other by a permutation of coordinates. The permutation $\sigma \in S_{n}$ is an automorphism of $\mathcal{C}$ if $\mathcal{C}=\mathcal{C} \sigma$. The set of all automorphisms of $\mathcal{C}$ forms the automorphism group $\operatorname{Aut}(\mathcal{C})$ of $\mathcal{C}$. In particular, when $n$ is even, the permutation $\tau \in S_{n}$ is called a fixed-point-free automorphism of order 2 if $\tau \in \operatorname{Aut}(\mathcal{C})$ and $\tau=\left(a_{1}, a_{2}\right)\left(a_{3}, a_{4}\right) \cdots\left(a_{n-1}, a_{n}\right)$, where $a_{i} \in\{1,2, \ldots, n\}$ and $a_{i} \neq a_{j}$ for $i \neq j$.

Let $\mathcal{C}$ be a binary self-dual code of length $2 n$ with a fixed-point-free automorphism $\sigma$ of order 2. We may assume that

$$
\sigma=(1,2)(3,4) \cdots(2 n-1,2 n) .
$$

Denote the cycles of $\sigma$ by $\Omega_{1}, \Omega_{2}, \ldots, \Omega_{n}$. Let $\mathbf{F}_{\sigma}(\mathcal{C})=\{\mathbf{v} \in \mathcal{C} \mid \mathbf{v} \sigma=\mathbf{v}\}$ and $\mathbf{E}_{\sigma}(\mathcal{C})=\left\{\mathbf{v} \in \mathcal{C} \mid w t\left(\mathbf{v} \mid \Omega_{i}\right)=0\right.$ or $\left.1, \forall i=1,2, \ldots, n\right\}$, where $\mathbf{v} \mid \Omega_{i}$ is the restriction of $\mathbf{v}$ on $\Omega_{i}$. 
A method for constructing binary self-dual codes with a fixed-point-free automorphism of order 2 is given in [5]. The basis of this method is the following proposition.

Proposition 2.2 ([5, Theorem $])$. Let $\mathcal{C}^{\prime}$ be a self-orthogonal $\left[k, s, d^{\prime}\right]$ code, $\mathcal{C}^{\prime \prime}$ be its dual code and $\psi: \mathcal{C}^{\prime \prime} \rightarrow \mathbb{F}_{2}^{2 k}$ be the map defined by $\psi(\mathbf{u})=\left(\alpha_{1}, \alpha_{1}, \ldots, \alpha_{k}\right.$, $\left.\alpha_{k}\right)$ for $\mathbf{u}=\left(\alpha_{1}, \ldots, \alpha_{k}\right) \in \mathcal{C}^{\prime \prime}$. Let $M=\left\{\left(j_{1}, j_{2}\right),\left(j_{3}, j_{4}\right), \ldots,\left(j_{2 r-1}, j_{2 r}\right)\right\}$ be a set of $r$ pairs of different coordinates of the code $\mathcal{C}^{\prime}, 0 \leq 2 r \leq k$, and $\varphi: \mathcal{C}^{\prime} \rightarrow \mathbb{F}_{2}^{2 k}$ be the map defined by $\varphi(\mathbf{u})=\left(\alpha_{1}^{\prime}, \alpha_{1}^{\prime \prime}, \ldots, \alpha_{k}^{\prime}, \alpha_{k}^{\prime \prime}\right)$ for $\mathbf{u}=$ $\left(\alpha_{1}, \alpha_{2}, \ldots, \alpha_{k}\right) \in \mathcal{C}^{\prime}$, where $\left(\alpha_{i}^{\prime}, \alpha_{i}^{\prime \prime}\right)=\left(\alpha_{i}, 0\right)$ for $i \neq j_{l}, l=1,2, \ldots, 2 r$, and $\left(\alpha_{2 i-1}^{\prime}, \alpha_{2 i-1}^{\prime \prime}, \alpha_{2 i}^{\prime}, \alpha_{2 i}^{\prime \prime}\right)$ is given in Table 1. Then $\mathcal{C}=\varphi\left(\mathcal{C}^{\prime}\right)+\psi\left(\mathcal{C}^{\prime \prime}\right)$ is a selfdual $[2 k, k, d]$ code with $\min \left\{d^{\prime}, 2 d^{\prime \prime}\right\} \leq d \leq 2 d^{\prime \prime}$. Moreover, the code $\mathcal{C}$ has an automorphism $\sigma$ defined in (1).

TABLE 1. Evaluation

\begin{tabular}{|c|c||c|c|}
\hline$\left(\alpha_{j_{2 i-1}}, \alpha_{j_{2 i}}\right)$ & $\left(\alpha_{j_{2 i-1}}^{\prime}, \alpha_{j_{2 i-1}}^{\prime \prime}, \alpha_{j_{2 i}}^{\prime}, \alpha_{j_{2 i}}^{\prime \prime}\right)$ & $\left(\alpha_{j_{2 i-1}}, \alpha_{j_{2 i}}\right)$ & $\left(\alpha_{j_{2 i-1}}^{\prime}, \alpha_{j_{2 i-1}}^{\prime \prime}, \alpha_{j_{2 i}}^{\prime}, \alpha_{j_{2 i}}^{\prime \prime}\right)$ \\
\hline$(0,0)$ & $(0,0,0,0)$ & $(1,0)$ & $(1,0,1,1)$ \\
$(1,1)$ & $(0,1,0,1)$ & $(0,1)$ & $(1,1,1,0)$ \\
\hline
\end{tabular}

\subsection{Length 50}

All possible weight enumerators for length 50 are

$$
W_{50,1}=1+196 y^{10}+11368 y^{12}+31752 y^{14}+\cdots
$$

and

$$
W_{50,2}=1+(580-32 \beta) y^{10}+(7400+160 \beta) y^{12}+\cdots,
$$

where $\beta$ is an integer parameter, $0 \leq \beta \leq 2$ (see $[7,14])$.

We construct two new binary optimal self-dual $[50,25,10]$ codes with a fixed point free automorphism of order 2 . We use a self-orthogonal $[25,12,6]$ code with a generator matrix $G_{25}=\left(I_{12} B\right)$ where $I_{12}$ is the identity matrix, and $B$ is the $12 \times 12$ circulant matrix with the first row vector 1100101111101 . We let $r=10$ and $M=\{(1,4),(2,20),(3,19),(5,21),(6,7),(8,9),(10,11),(17,18)$, $(22,23),(24,25)\}$, then we obtain a self-dual $[50,25,10]$ code $\mathcal{C}_{50,1}$ with weight enumerator $W_{2}$ for $\beta=0$. The order of the automorphism group of $\mathcal{C}_{50,1}$ is 2 . If we let $r=11$ and $M=\{(1,7),(2,12),(3,13),(6,8),(9,10),(14,15),(16,17)$, $(18,19),(20,21),(22,23),(24,25)\}$, then we obtain an optimal self-dual code $[50,25,10]$ code $\mathcal{C}_{50,2}$ with weight enumerator $W_{2}$ for $\beta=0$. The order of the automorphism group of $\mathcal{C}_{50,2}$ is 2 . These two codes are inequivalent; they are also inequivalent to the two codes, which we denote by $\mathcal{C}_{50,3}$ and $\mathcal{C}_{50,4}$, with $\beta=0$ that possess a fixed-point-free automorphism of order 2 found in [3].

Theorem 2.3. There exist at least four inequivalent binary self-dual [50, 25, 10] codes with a fixed-point-free automorphism of order 2 . These codes have automorphism groups of order 2 . 
The matrix $G_{50,1}$ (respectively, $G_{50,2}, G_{50,3}, G_{50,4}$ ) is a generator matrix of the code $\mathcal{C}_{50,1}$ (respectively, $C_{50,2}, C_{50,3}, C_{50,4}$ ).

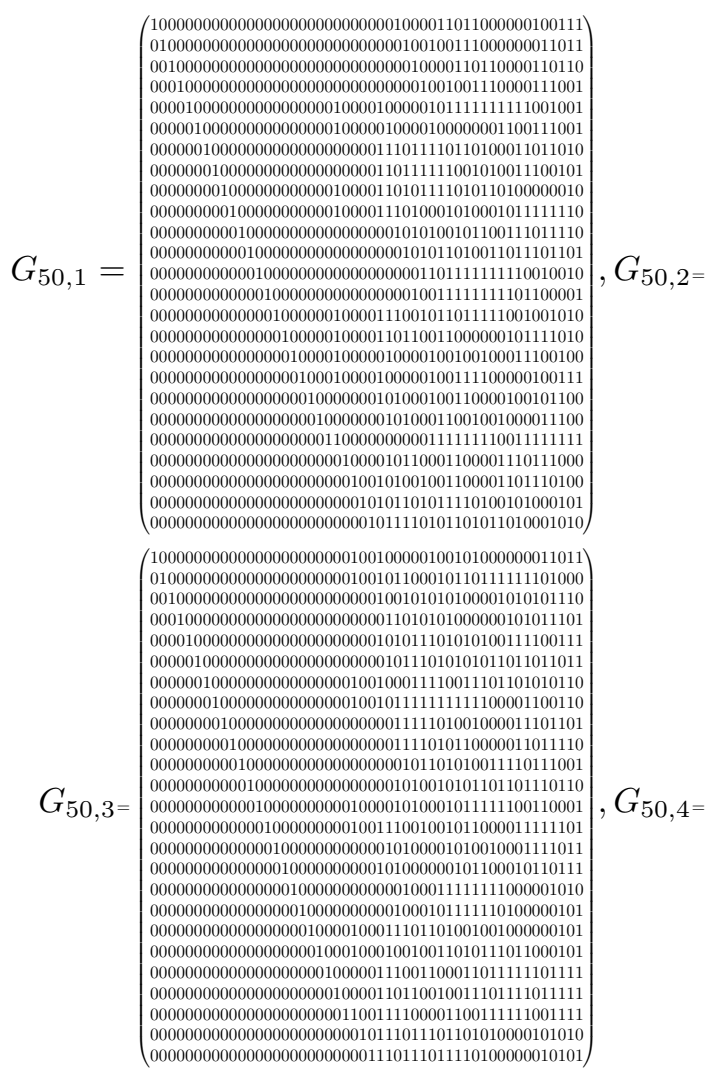

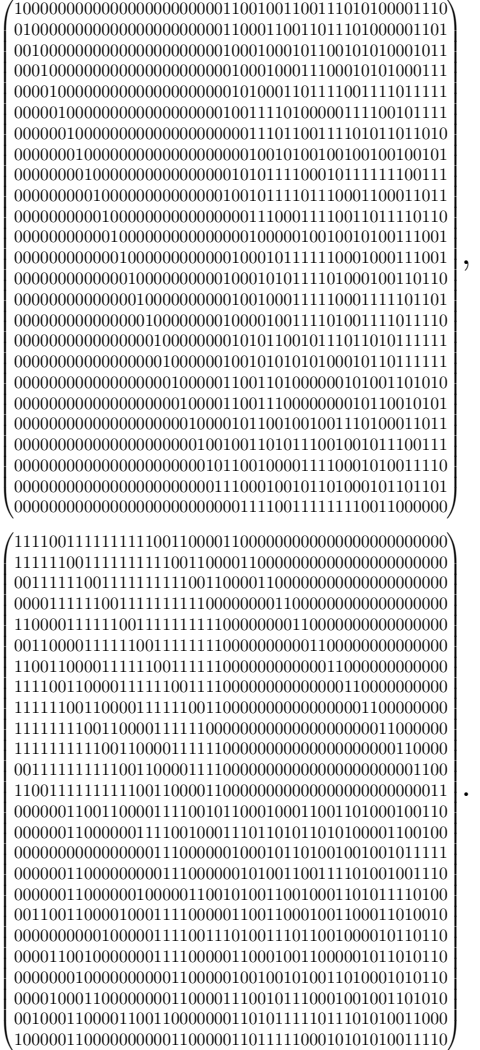

\subsection{Extension}

The following lemma enables to construct a self-dual code of length $2 n+2$ from a self-dual code of length $2 n$.

Lemma 2.4 ([26, Theorem 1]). Let $G_{0}$ be a generator matrix of a self-dual code $\mathcal{C}_{0}$ of length $2 n$, and let

$$
\mathbf{x}=\left(x_{1}, \ldots, x_{n}, x_{n+1}, \ldots, x_{2 n}\right)
$$

be a vector in $\mathbf{F}_{2}^{2 n}$ such that $\mathbf{x} \cdot \mathbf{x}=1$, where $\cdot$ denotes the Euclidean inner product. Let

$$
y_{i}:=\mathbf{x} \cdot \mathbf{r}_{i}
$$


for $1 \leq i \leq n$, where $\mathbf{r}_{i}$ is the $i$-th row vector of $G_{0}$. Then the following matrix

$$
G=\left(\begin{array}{cc|cccc}
1 & 0 & x_{1} & x_{2} & \cdots & x_{2 n} \\
\hline y_{1} & y_{1} & & & & \\
\vdots & \vdots & & & G_{0} & \\
y_{n} & y_{n} & & & &
\end{array}\right)
$$

generates a self-dual code $\mathcal{C}$ of length $2 n+2$.

The extension method is initially used by Harada in 1997 [13]. For odd prime $p$, we find the construction method for a self-dual code of length $2 n+2$ with an automorphism of order $p$ with $c$ independent cycles from a self-dual code of length $2 n$ with an automorphism of order $p$ with $c$ independent cycles in [23]. The following theorem is an extension of a self-dual code which has a fixedpoint-free automorphism $\bar{\sigma}$ of order 2 preserving the order of an automorphism. We may assume that

$$
\bar{\sigma}=(1,2)(3,4) \cdots(2 n+1,2 n+2) .
$$

Theorem 2.5. Let $G$ be a generator matrix of a self-dual code $\mathcal{C}$ of length $2 n$ with a fixed-point-free automorphism $\sigma$ defined in (1). Suppose that $\mathbf{v}$ is a vector $\mathbf{F}_{\sigma}(\mathcal{C})$ with its weight is not divisible by four, and $\mathbf{v}_{1}=\left(v_{1}, v_{2}, \ldots, v_{2 n}\right)$ is a vector in $\mathbb{F}_{2}^{2 n}$ with $\mathbf{v}=\mathbf{v}_{1}+\mathbf{v}_{1} \sigma$. Then the following matrix

$$
\bar{G}=\left(\begin{array}{cc|llll}
1 & 0 & v_{1} & v_{2} & \cdots & v_{2 n} \\
\hline y_{1} & y_{1} & & & & \\
\vdots & \vdots & & & G & \\
y_{n} & y_{n} & & & &
\end{array}\right)
$$

generates a self dual $\overline{\mathcal{C}}$ of length $2 n+2$ with a fixed-point-free automorphism $\bar{\sigma}$ defined in (2), where $y_{i}:=\mathbf{v}_{1} \cdot \mathbf{r}_{i}$ for $1 \leq i \leq n$, where $\mathbf{r}_{i}$ is the $i$-th row vector of $G$.

Proof. By hypothesis, we have $w t\left(\mathbf{v}_{1} \mid \Omega_{i}\right)=w t\left(\mathbf{v}_{1} \sigma \mid \Omega_{i}\right)$ for $i=1,2, \ldots, n$. Since the weight of $\mathbf{v}$ is not divided by four, the weight of $\mathbf{v}_{1}$ is odd. The matrix $\bar{G}$ generates a self-dual code $\overline{\mathcal{C}}$ of length $2 n+2$ by Theorem 2.4. Since $\mathcal{C}$ has an automorphism $\sigma$, it is sufficient to show that $\left(1,0, \mathbf{v}_{1}\right) \bar{\sigma}$ is in $\overline{\mathcal{C}}$. As $\mathbf{v} \in \mathcal{C}$, there exists $y \in\{0,1\}$ such that $(y, y, \mathbf{v}) \in \overline{\mathcal{C}}$. Since $\mathbf{v}_{1}$ has odd weight, $\mathbf{v}_{1} \notin \mathcal{C}=\mathcal{C}^{\perp}$, implying that $\mathbf{v}_{1} \cdot \mathbf{v}=1$. Since $\overline{\mathcal{C}}$ is self-dual, $0=(y, y, \mathbf{v}) \cdot\left(1,0, \mathbf{v}_{1}\right)=y+1$. Thus $(1,1, \mathbf{v}) \in \overline{\mathcal{C}}$. We have that $\left(1,0, \mathbf{v}_{1}\right) \bar{\sigma}=\left(0,1, \mathbf{v}_{1} \sigma\right)=(1,1, \mathbf{v})+\left(1,0, \mathbf{v}_{1}\right) \in \overline{\mathcal{C}}$. Therefore, the code $\overline{\mathcal{C}}$ has the automorphism $\bar{\sigma}$.

Theorem 2.5 shows that we can construct a self-dual code of length $2 n+2$ with a fixed-point-free automorphism of order 2 from a self-dual code of length $2 n$ with a fixed-point-free automorphism of order 2 .

Corollary 2.6. Suppose that $n$ is odd. If there exists a self-dual code of length $2 n$ with a fixed-point-free automorphism of order 2 , then there exists a self-dual code of length $2 n+2$ with a fixed-point-free automorphism of order 2 . 
Proof. Let $\mathcal{C}$ be a self-dual code of length $2 n$ with a fixed-point-free automorphism of order 2, where $n$ is odd. Since the code $\mathcal{C}$ is self-dual, it has the all ones vector. The weight of the all one vector is not divided by four since $n$ is odd. By Theorem 2.5, there exists a self-dual code of length $2 n+2$ with a fixed-point-free automorphism of order 2.

We may assume that

$$
\widetilde{\sigma}=(1,2)(3,4) \cdots(2 n-3,2 n-2) .
$$

The following theorem shows that we can construct a self-dual code of length $2 n-2$ with a fixed-point-free automorphism $\widetilde{\sigma}$ of order 2 from a self-dual code of length $2 n$ with a fixed-point-free automorphism $\sigma$ of order 2 . This means that all self-dual codes of length $2 n$ with a fixed-point-free automorphism of order 2 can be obtained from self-dual codes of length $2 n-2$ with a fixed-point-free automorphism of order 2 by adding a suitable row and corresponding columns.

Theorem 2.7. Let $\mathcal{C}$ be a binary self-dual $[2 n, n, d]$ code with a fixed-point-free automorphism of order 2 . Then $\mathcal{C}$ is constructed from some self-dual code of length $2 n-2$ with a fixed-point-free automorphism of order 2 .

Proof. Let $\mathcal{C}$ be a binary self-dual code of length $2 n$ with an automorphism $\sigma$ defined in (1) and let $G$ be the generator matrix of $\mathcal{C}$ in reduced row echelon form. First, we suppose that $G$ is of the form

$$
\left(\begin{array}{ccccc}
1 & 0 & * & \cdots & * \\
0 & 1 & * & \ldots & * \\
0 & 0 & * & \ldots & * \\
\vdots & \vdots & & \vdots & \\
0 & 0 & * & \cdots & *
\end{array}\right) .
$$

We add the first row vector of the matrix $G$ to its second row vector and then delete the first row vector, the first column, and the second column. As a result, we obtain the $(n-1) \times(2 n-2)$ matrix denoted by $\widetilde{G}$. Since the code $\mathcal{C}$ is self-dual and the matrix $G$ is in reduced row echelon form, the rank of the matrix $\widetilde{G}$ is $n-1$. Hence $\widetilde{G}$ generates a self-dual code of length $2 n-2$. Let $\mathbf{r}_{i}$ be the $i$-th row vector of $G$. Since $\mathcal{C}$ has the automorphism $\sigma$ and $G$ is in reduced row echelon form, we have $\mathbf{r}_{1} \sigma=\mathbf{r}_{2}$. Hence $\left(\mathbf{r}_{1}+\mathbf{r}_{2}\right) \sigma=\mathbf{r}_{1}+\mathbf{r}_{2}$. Therefore, the code generated by $\widetilde{G}$ is a self-dual code of length $2 n-2$ with an automorphism $\tilde{\sigma}$ defined in (4). In the other case, we suppose that $G$ is of the form

$$
\left(\begin{array}{cccccc}
1 & 1 & 0 & * & \cdots & * \\
0 & 0 & 1 & * & \cdots & * \\
0 & 0 & 0 & * & \cdots & * \\
\vdots & \vdots & \vdots & & \vdots & \\
0 & 0 & 0 & * & \cdots & *
\end{array}\right) .
$$


We delete the first row vector of the matrix $G$. As a result, we obtain the $(n-1) \times 2 n$ matrix $G^{\prime}$. Since $G$ is in reduced row echelon form and $\mathcal{C}$ has the automorphism $\sigma$, the first row vector of $G$ is in $\mathbf{F}_{\sigma}(\mathcal{C})$. Hence the matrix $G^{\prime}$ generates a self-orthogonal code with the automorphism $\sigma$. Let $\widetilde{G}$ be the matrix obtained by deleting the first and second columns of $G^{\prime}$. Then $\widetilde{G}$ generates a self-dual code of length $2 n-2$ with an automorphism $\widetilde{\sigma}$ defined in (4).

\subsection{Length 52}

All possible weight enumerators for length 52 are

$$
W_{52,1}=1+250 y^{10}+7980 y^{12}+4800 y^{14}+\cdots,
$$

and

$$
W_{52,2}=1+(442-16 \beta) y^{10}+(6188+64 \beta) y^{12}+53040 y^{14}+\cdots
$$

for $\beta=0,1, \ldots, 10$, or 12 (see $[6,7])$.

We construct 29 (respectively, 87, 23, 45) inequivalent binary self-dual $[52,26,10]$ codes with a fixed-point-free automorphism of order 2 from $\mathcal{C}_{50,1}$ (respectively, $\mathcal{C}_{50,2}, \mathcal{C}_{50,3}, \mathcal{C}_{50,4}$ ) by Theorem 2.5. Since they have automorphism groups of order 2 , we check the equivalence between them and the codes in $[3,7]$. We find eight inequivalent optimal self-dual codes of length 52 with a fixed-point-free automorphism of order 2 in [3, 7]. We obtain the following result.

Theorem 2.8. There exist at least 184 inequivalent binary self-dual [52, 26, 10] codes with a fixed-point-free automorphism of order 2 . All of these codes have automorphism groups of order 2 .

In Table 2, we list the results which are obtained from $\mathcal{C}_{50,1}$ in hexadecimal number.

\begin{tabular}{|c|c|c|c|c|c|}
\hline $\mathbf{v}_{1}$ & $\beta$ & $\mathbf{v}_{1}$ & $\bar{\beta}$ & $\mathbf{v}_{1}$ & $\beta$ \\
\hline 8 a a a a a 882 a 80 & 2 & a a 2 a a 20208882 & 1 & 88828 a 802 a 8 a 2 & 2 \\
\hline 2 a a a a 20 a a 02 & 2 & 820 a a a 8880 a 22 & 2 & a 02 a a 800 a 2 a 22 & 1 \\
\hline 2 a 28 a a a 282822 & 2 & 882 a 2802 a 8 a 82 & 0 & a a a 808208 a 0 a 2 & 0 \\
\hline 282 a 28 a a a 022 & 0 & 0 a a 82 a 8 a 200 a 2 & 0 & 28 a 2 a a 0 a 2 a 200 & 2 \\
\hline 82 a a 2 a a 8 a 0 a 02 & 2 & 20 a a a 0 a 0 a 08 a 2 & 1 & a a 2 a 0 a 0 a 020 a 2 & 1 \\
\hline a a 28 a 2208 a 82 & 0 & a 02 a 888 a 80880 & 2 & 82 a a 2 a 0 a 2 a 020 & 4 \\
\hline a a 2888 a 88 a 222 & 1 & 82 a a 802820 a 20 & 0 & 28 a 8 a 2020 a a 0 & 2 \\
\hline 02 a a 0 a a a 2822 & 1 & a 8800 a 0 a 8 a 820 & 0 & 0 a a 080 a a a 820 & 1 \\
\hline a 8 a 828 a 2822 a 2 & 4 & 2882222022 a 22 & 0 & a 88 a 0028 a a 2 a 0 & 1 \\
\hline 2002 a a 2 a 282 a 2 & 0 & a 280882882 a 20 & 0 & & \\
\hline
\end{tabular}

TABLE 2. Vectors $\mathbf{v}_{1}$ in Theorem 2.5 for $\mathcal{C}_{50,1}$

In Table 3, we list the results which are obtained from $\mathcal{C}_{50,2}$ in hexadecimal number. 
TABLE 3. Vectors $\mathbf{v}_{1}$ in Theorem 2.5 for $\mathcal{C}_{50,2}$

\begin{tabular}{|c|c|c|c|c|c|}
\hline $\mathbf{v}_{1}$ & $\beta$ & $\mathbf{v}_{1}$ & $\beta$ & $\mathbf{v}_{1}$ & $\beta$ \\
\hline 0 a a a 222800 a 20 & 0 & 8 a 0 a 8 a a 8282 a 2 & 1 & a 28222 a 222882 & 1 \\
\hline a 0 a 220 a 882280 & 1 & 22 a a 80 a 22 a a a 0 & 0 & a 8228 a a 88 a 020 & 0 \\
\hline 280 a 80 a a 0 a a 00 & 0 & 820 a a 0 a a 0 a a a 2 & 5 & a a 22 a 22202 a 80 & $W_{52,1}$ \\
\hline a 022880282 a 22 & 2 & 2 a а 2 а а 2 а 00 а 82 & 0 & 2282 a a a 80 a 220 & 0 \\
\hline a 280222 a 2 a 800 & 1 & a a 22 a a 882 a 220 & 2 & 80 a a a 000 a a a 82 & 0 \\
\hline a a a 00 a 000 a 2 а 0 & 0 & 82 a a a 22 a 808 a 2 & 1 & 2 a a 2 a 28028222 & 0 \\
\hline 288 a 800282 a a 0 & 1 & a 22 a a 20 a 8 a 222 & 1 & a a 8 a 28280 a 202 & 3 \\
\hline a a a 0280 a 80280 & 1 & 2828 a 8 a a a 20 a 2 & 2 & 8 a 08 a a 2222882 & 2 \\
\hline 220 a 802 a 28222 & 2 & a 8 a 22 a a 8 a 2022 & 0 & 880 a 8 a 8 a 8 a a 00 & 1 \\
\hline 22008 a 8 a 888 a 0 & 3 & a 8820 a 8 a 2 a 2 a 2 & 0 & a a 2 a 82 a a 08800 & 3 \\
\hline 88208 a a 800 a 82 & 1 & 28 a 2 a 2 а 28 a a 80 & 0 & a 8 a 808 a 0288 a 2 & 3 \\
\hline 282 a 088 a 0 a 082 & 1 & 22 a 00 a a 828 a 22 & 1 & a a 0 a 2880822 a 2 & 2 \\
\hline a 288 a 8 a 820800 & 3 & 2 a 0 a a 08 a a a 800 & 4 & 2882 a a 2828 a 02 & 1 \\
\hline 0 a 08 a 282 a 8820 & 0 & 8828 a 28 a 220 a 2 & 0 & 222 a 22 a a 8 a 020 & 0 \\
\hline a 00 a 20 a a 88220 & 3 & a 082 a 022 a a 0 a 2 & 0 & 8 a 2088822 a 8 a 2 & 0 \\
\hline 8808 a 80808 a a 2 & 0 & 22 а 028 a 2 а 2 а 02 & 0 & 082 a a 8 a a 28 a 00 & 0 \\
\hline a a 2008 a 00 a 882 & 2 & 2 a 0288 a 888220 & 3 & 80 a a 0 a 0 a a a 802 & 4 \\
\hline a 0 a 0 a 08 a 28802 & 2 & 020 a a 0 a 0 a 82 a 0 & 0 & 820 a 8 a 0 a a 8222 & 1 \\
\hline 202828 a 280 a 22 & 2 & 22 a a a 0 a 028002 & 1 & a 202 a a 0 a 20 a 82 & 2 \\
\hline a a 0 a 0028 a 80 a 0 & 3 & a a a 2002 a a 2000 & 3 & 80 a 208882 a a a 2 & 1 \\
\hline 08 a a a 0 a 888200 & 1 & 28 a 2 a 0 a a 02002 & 1 & 282 a 222 a a 8802 & 0 \\
\hline 2 a 288228 a 2080 & 0 & 0 a a 82 a 0 a 20202 & 1 & a 88 a 8 a a a 80080 & 1 \\
\hline a 802 a 2202 a a 00 & 0 & 0 a 22 a 80282 a 80 & 1 & 2088 a a 88 a 8222 & 0 \\
\hline 8 a a 00 a 8888880 & 1 & a 082208808 a a 2 & 0 & 002 a 2 a a a 82 a 02 & 1 \\
\hline 802082 a 0 a 82 a 2 & 3 & 88 a 8888208882 & 3 & 0 a a a 282 a 2020 & 0 \\
\hline 28 a 0888 a 20282 & 2 & a a 200 a a 882200 & 2 & 82 a a a 88820 a 80 & 0 \\
\hline a 802 a 028 a 2082 & 0 & a a 082 a 00028 a 2 & 2 & 2 a 0 a a 28222282 & 1 \\
\hline 8 a a 8 a 8 a 2 a 8 a 82 & 1 & a 2002282 a 28 a 0 & 0 & a 820828 a a a 802 & 5 \\
\hline a a a 80 a 8 a 02 a 82 & 2 & 20 a 288 a 0 a 8280 & & a 2 a 8 a 02282882 & 0 \\
\hline
\end{tabular}

In Table 4 , we list the results which are obtained from $\mathcal{C}_{50,3}$ in hexadecimal number.

TABLE 4. Vectors $\mathbf{v}_{1}$ in Theorem 2.5 for $\mathcal{C}_{50,3}$

\begin{tabular}{|c|c|c|c|c|c|}
\hline $\mathbf{v}_{1}$ & $\beta$ & $\mathbf{v}_{1}$ & $\beta$ & $\mathbf{v}_{1}$ & $\beta$ \\
\hline 2 a a 8 a 0 a 8 a a 8 a 2 & 1 & 8 a a 8 a 2 a 2 a 2280 & 2 & 2 a 80 a а 2 a 28 a a 0 & 1 \\
\hline a 2 a 2208 a 22 a a 2 & 0 & 2 a 28 a 8 a 2808 a 0 & 2 & 28 a a 2 a 2 a 0 a 020 & 1 \\
\hline a a 2 a 22 a 800282 & 1 & 2 a 2 a a 80220 a 22 & 0 & 828 a 2228822 a 2 & 1 \\
\hline a 0 a 820882 a a 22 & 1 & a 828 a 82 a a 0 a 00 & 0 & 80 a a a 08220 a a 2 & 0 \\
\hline 220882 a 8 a a a 80 & 1 & 0 a 2828082 a a a 2 & 0 & 2 a 88 a 82008 a a 2 & 1 \\
\hline 0 a 82 a a a 0282 a 0 & 2 & 2 a 0 a 8 a 2 a 22022 & 2 & 828 a 880828222 & 2 \\
\hline 0 a a a 0 a a 20000 & 0 & 208 a 228 a 02 a 80 & 0 & 0 a 880 a a 80 a 082 & 1 \\
\hline 0228 a 02282 a a 0 & 1 & 0 a a 0802208 a a 2 & 0 & & \\
\hline
\end{tabular}

In Table 5, we list the results which are obtained from $\mathcal{C}_{50,4}$ in hexadecimal number. 
TABLE 5. Vectors $\mathbf{v}_{1}$ in Theorem 2.5 for $\mathcal{C}_{50,4}$

\begin{tabular}{|c|c|c|c|c|c|}
\hline $\mathbf{v}_{1}$ & $\beta$ & $\mathbf{v}_{1}$ & $\beta$ & $v$ & \\
\hline 222 a а 88 a 22 a 82 & 3 & 28 a a a 0 a a a 2880 & 2 & 8 a a 2882 a a 02 a 2 & 4 \\
\hline 82802 a a a 2 a a 22 & 2 & а 288 a 88 a 2 a 2 a 0 & 5 & 8 a a 2288 a a a 220 & 3 \\
\hline 2 a 0822 a 2 a a a 22 & 2 & 2 a a 2 a 08 a 0 a a a 0 & 3 & 8 a a 8 a a a 802822 & 3 \\
\hline 28088 a a a 88 a a 2 & 3 & a a 822 a 828 a 200 & 3 & 2 a a 0282888 a 22 & 2 \\
\hline 08 a 82 a a 8 a 8 a 00 & 2 & a 208 a 8288 a a 02 & 3 & a a a a 8 a 2002022 & 3 \\
\hline 0 a 0 a 2 а 82 a 8 a 02 & 4 & a a 0 a a 8 a 808880 & 4 & 82 a 08 a a 288882 & 2 \\
\hline 02 а 28 a а 888 a 20 & 3 & 2828 a 2 a a 220 a 0 & 2 & a 82888 a a 00 a a 0 & 2 \\
\hline a a a 0 a 288 a 00 a 0 & 2 & a 2082 a 0 a 828 a 2 & 2 & 282220888 a a a 2 & 3 \\
\hline 8 a 20 a 282222 a 2 & 2 & a a 802820 a a 282 & 2 & a 8 a 8 a 2 a 808280 & 3 \\
\hline а 2 а 28 a a 020 a 02 & 3 & 8 a 000 a a 82 a 2 a 2 & 2 & 28808 a a 202282 & 4 \\
\hline 88088 a a 220 a 80 & 2 & 2 a 0228880 a 882 & 3 & 20008 a 2 a a a 082 & 3 \\
\hline 0800 a 82 a 822 a 2 & 2 & 0882808 a a a a 00 & 4 & a 80 a 808 a 88202 & 2 \\
\hline 2 a 2 a 0 a 088 a 800 & 2 & 28 a 8 a 20882802 & 2 & 28 a a 0 a 20 a 8200 & 6 \\
\hline 28 a 022880 a 282 & 2 & 08828 a a 0 a a 002 & 2 & a a 20 a a 08 a a a a 2 & 2 \\
\hline 2 a a a 0 a a a 2 a 0 a 2 & 5 & a 0 a 8 a a a 0 a a 82 & 3 & 2 a 2 a a 28 a 2 a a 22 & \\
\hline
\end{tabular}

\section{Self-dual code over $\mathbb{F}_{2}+u \mathbb{F}_{2}$}

The ring $\mathbb{F}_{2}+u \mathbb{F}_{2}$ is a finite ring of 4 elements with a nilpotent element $u$ where $u^{2}=0$. In this ring $\mathbb{F}_{2}+u \mathbb{F}_{2}$, addition is defined by $(a+b u)+(c+d u)=$ $(a+c)+(b+d) u$ and multiplication by $(a+b u)(c+d u)=a c+(a d+b c) u$ where $a, b, c, d \in \mathbb{F}_{2}$. We denote $1+u$ by $v$. The units are 1 and $v$ in $\mathbb{F}_{2}+u \mathbb{F}_{2}$. If $\mathcal{R}=\mathbb{F}_{2}+u \mathbb{F}_{2}$, then a linear code $\mathcal{C}$ of length $n$ over $\mathcal{R}$ is an $\mathcal{R}$-submodule of $\mathcal{R}^{n}$. We consider self-dual codes over $\mathcal{R}$. We define the ordinary inner product of $\mathbf{x}$ and $\mathbf{y}$ in $\mathcal{R}^{n}$ by $\langle\mathbf{x}, \mathbf{y}\rangle=\sum_{i=1}^{n} x_{i} y_{i}$, where $\mathbf{x}=x_{1} x_{2} \cdots x_{n}$ and $\mathbf{y}=y_{1} y_{2} \cdots y_{n}$ are in $\mathcal{R}^{n}$. The dual code of $\mathcal{C}$ is $\mathcal{C}^{\perp}=\left\{\mathbf{x} \in \mathcal{R}^{n} \mid\langle\mathbf{x}, \mathbf{y}\rangle=0\right.$ for all $\left.\mathbf{y} \in \mathcal{C}\right\}$. We say that $\mathcal{C}$ is self-orthogonal if $\mathcal{C} \subseteq \mathcal{C}^{\perp}$ and self-dual if $\mathcal{C}=\mathcal{C}^{\perp}$. We consider two weights as follows. For $\mathbf{x} \in \mathcal{R}^{n}$, the Hamming weight $\mathrm{wt}_{\mathrm{H}}(\mathbf{x})$ is the number of non-zero components of $\mathbf{x}$. The Lee weights of the elements $0,1, u, v$ of $\mathcal{R}$ are $0,1,2$, and 1 , respectively, and the Lee weight $\mathrm{wt}_{\mathrm{L}}(\mathbf{x})$ is the sum of the Lee weights of components of $\mathbf{x}$ [1]. The minimum Lee (respectively, Hamming) weight of a code $\mathcal{C}$ is the smallest nonzero Lee (respectively, Hamming) weight of a codeword in $\mathcal{C}$. Let $\mathcal{M}_{n}$ be the set of $n \times n$ invertible monomial matrices over $\mathcal{R}$. Two codes $\mathcal{C}_{1}$ and $\mathcal{C}_{2}$ of length $n$ are monomially equivalent if $\mathcal{C}_{2}=\mathcal{C}_{1} M$ for some $M \in \mathcal{M}_{n}$. The monomial automorphism group of $\mathcal{C}$ is $\operatorname{Aut}(\mathcal{C})=\{M \in$ $\left.\mathcal{M}_{n} \mid \mathcal{C} M=\mathcal{C}\right\}$.

\subsection{Strategy}

We define the Gray map $\mathfrak{G}$ as follows [9]:

$$
\mathfrak{G}: \mathcal{R} \rightarrow \mathbb{F}_{2}^{2} \text { by } \mathfrak{G}(0)=00, \mathfrak{G}(1)=10, \mathfrak{G}(v)=01 \text {, and } \mathfrak{G}(u)=11 .
$$

When $\mathbf{x}$ is in $\mathcal{R}^{n}$, we apply $\mathfrak{G}$ to each component of $\mathbf{x}$. For a code $\mathcal{C}$ of length $n$ over $\mathcal{R}$, the binary code $\mathfrak{G}(\mathcal{C})$ of length $2 n$ is called the Gray image of $\mathcal{C}$. This map is $\mathbb{F}_{2}$-linear, and so if $\mathcal{C}$ is a linear code of length $n$ over $\mathcal{R}$, then $\mathfrak{G}(\mathcal{C})$ is a binary linear code of length $2 n[18]$. We have $\operatorname{wt}_{\mathbf{L}}(\mathbf{x})=\mathrm{wt}(\mathfrak{G}(\mathbf{x}))$, where wt denotes the ordinary Hamming weight of a binary vector. In fact, if the Lee 
distance between vectors $\mathbf{x}$ and $\mathbf{y}$ is $\mathrm{wt}_{\mathrm{L}}(\mathbf{x}-\mathbf{y})$, then $\mathfrak{G}$ is an isometry from $\mathcal{R}^{n}$ under the Lee distance to $\mathbb{F}_{2}^{2 n}$ under the Hamming distance [9].

We concisely explain the $\tau$-method which is introduced in $[1,19]$ as follows. Let $\mathcal{C}$ be a self-dual code over $\mathbb{F}_{2}+u \mathbb{F}_{2}$ of length $n$. If $\mathcal{C}$ has minimum Lee weight $d_{L}$, then the Gray image of $\mathcal{C}$ is a binary self-dual $\left[2 n, n, d_{L}\right]$ code having a fixed-point-free automorphism $\tau=(1,2)(3,4) \cdots(2 n-1,2 n)$. Conversely, for a binary self-dual $[2 n, n, d]$ code $\mathcal{D}$ having a fixed-point-free automorphism $\tau^{\prime}=\left(a_{1}, b_{1}\right)\left(a_{2}, b_{2}\right) \cdots\left(a_{n}, b_{n}\right)$ of order 2 , we can find an equivalent code $\mathcal{D}^{\prime}$ by rearranging the coordinates of $\mathcal{D}$ in the order of $a_{1}, b_{1}, a_{2}, b_{2}, \ldots, a_{n}, b_{n}$. Then $\mathcal{D}^{\prime}$ has the fixed-point-free automorphism $\tau=(1,2)(3,4) \cdots(2 n-1,2 n)$, and $\mathfrak{G}^{-1}\left(\mathcal{D}^{\prime}\right)$ is a self-dual code over $\mathcal{R}$ with minimum Lee weight $d_{L}=d$. If $\mathcal{D}_{1}$ and $\mathcal{D}_{2}$ are two inequivalent binary self-dual codes with fixed-pointfree automorphisms of order 2 , then the preimages $\mathfrak{G}^{-1}\left(\mathcal{D}_{1}^{\prime}\right)$ and $\mathfrak{G}^{-1}\left(\mathcal{D}_{2}^{\prime}\right)$ are monomially inequivalent to each other. For a binary self-dual code $\mathcal{D}$ with two fixed-point-free automorphisms, $\tau_{1}$ and $\tau_{2}$, of order 2 , we obtain $\mathcal{D}_{1}^{\prime}$ and $\mathcal{D}_{2}^{\prime}$ by rearranging the coordinates of $\mathcal{D}$ with $\tau_{1}$ and $\tau_{2}$ respectively. Then $\mathfrak{G}^{-1}\left(\mathcal{D}_{1}^{\prime}\right)$ and $\mathfrak{G}^{-1}\left(\mathcal{D}_{2}^{\prime}\right)$ are monomially equivalent if and only if $\tau_{1}$ and $\tau_{2}$ are conjugate in the automorphism group of $\mathcal{D}$.

Our strategy for the classification of the self-dual codes over $\mathcal{R}$ of length $n$ is as follows [22]:

I. For a binary self-dual code $\mathcal{C}$ of length $2 n$, find all fixed-point-free automorphisms of order 2 .

II. Find all distinct conjugacy classes of fixed-point-free automorphisms of order 2 obtained in the step I.

III. Rearrange the coordinates of $\mathcal{C}$ by distinct fixed-point-free automorphisms in the step II, so that they have a fixed-point-free automorphism $\tau=(1,2)(3,4) \cdots(2 n-1,2 n)$.

V. By finding the preimage of the codes under $\mathfrak{G}$, we obtain inequivalent codes over $\mathbb{F}_{2}+u \mathbb{F}_{2}$.

We construct Lee-optimal self-dual codes over $\mathcal{R}$ of lengths 25 and 26 from the codes that were obtained in Section 2. We apply the $\tau$-method and our strategy to $\mathcal{C}_{50, i}$ for $i=1,2,3,4$. We present the generator matrices of the results as follows:

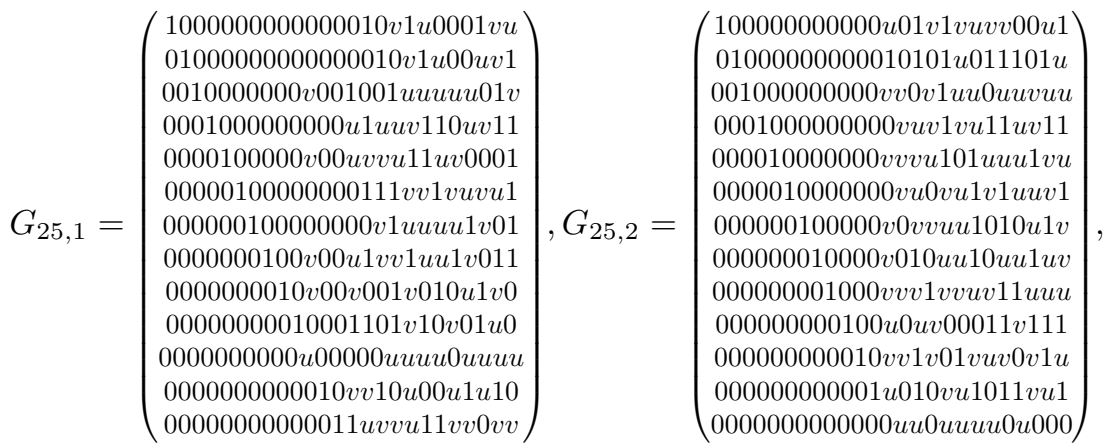




$$
G_{25,3}=\left(\begin{array}{c}
10000000000 v 01001 v v 000 v 1 u \\
01000000000001 v v v v 00111 u 1 \\
001000000000011 u 1111 v u 1 v u \\
00010000000 v 010 u u 0 u 1 u v v v 1 \\
00001000000000 u u 11 v 00 u 1 u v \\
00000100000000 v v 111 v u 1 u 1 v \\
00000010000 v 001101 u u u 0 u 0 v \\
0000000100000 v v 0011 v 0 v u 1 u \\
00000000100000 v 0 v u u u 10011 \\
00000000010 v 0 v u v 11 v 0100 v v \\
0000000000100 u 1 v 10 u v u u 1 u u \\
00000000000 u 0 u u 00 u 0 u u u 0 u u \\
0000000000001 u 1 u 1 u v v 00111
\end{array}\right), G_{25,4}=\left(\begin{array}{c}
100000000000 v 1 u u 1 u v v v 1 v 0 v \\
010000000000 v 1 v 0 u 10 v v 1 v v u \\
0010000000000 v 11 u u v u v v 111 \\
000100000000 v 01 v v u 01 u v v 1 v \\
000010000000 v 1 u 1 v v u 01 u v v v \\
000001000000 v v 1 u 1 v v 0 u 1 u 11 \\
000000100000 v 1 v 1 u 1 v 1001 u v \\
000000010000 v v 11 v u 111 u u v u \\
0000000010000 v v 1 v v 0111001 \\
000000000100 v u 11 \text { vvvuv1vu0} 0 \\
000000000010010 v 1111 u 1 v 1 u \\
00000000000100 v u v v v 1101 v v \\
000000000000 u 00 u 0 u u u u u 0 u u
\end{array}\right) .
$$

Theorem 3.1. There exist at least four inequivalent Lee-optimal self-dual codes over $\mathbb{F}_{2}+u \mathbb{F}_{2}$ of length 25 .

We apply the $\tau$-method and our strategy from the results of Theorem 2.8. In this case, we obtain 184 Lee-optimal self-dual codes over $\mathcal{R}$ of length 26 since codes have automorphism groups of order 2 .

Theorem 3.2. There are at least 184 inequivalent Lee-optimal self-dual codes over $\mathbb{F}_{2}+u \mathbb{F}_{2}$ of length 26 .

Remark 3.3. Since all codes that were obtained in Section 2 have automorphism groups of order 2, the result in this section can not be obtained by the decomposition theory of self-dual codes over $\mathbb{F}_{2}+u \mathbb{F}_{2}[18]$.

\section{References}

[1] E. Bannai, M. Harada, A. Munemasa, T. Ibukiyamasa, and M. Oura, Type II codes over $\mathbb{F}_{2}+u \mathbb{F}_{2}$ and applications to Hermitian modular forms, Abh. Math. Sem. Univ. Hamburg 73 (2003), 13-42.

[2] A. Bonnecaze, P. Sole, and A. R. Calderbank, Quaternary quadratic residue codes and unimodular lattices, IEEE Trans. Inform. Theory 41 (1995), no. 2, 366-377.

[3] S. Buyuklieva, New binary extremal self-dual codes with lengths 50 and 52, Serdica Math. J. 12 (1999), no. 3, 185-190.

[4] _ A method for constucting self-dual codes with an automorphism of order 2, IEEE Trans. Inform. Theory 46 (2000), no. 2, 496-504.

[5] S. Buyuklieva and I. Boukliev, Extremal self-dual codes with an automorphism of order 2, IEEE Trans. Inform. Theory 44 (1998), no. 1, 323-328.

[6] S. Buyuklieva, M. Harada, and A. Munemasa, Restrictions on the weight enumerators of binary self-dual codes of length $4 \mathrm{~m}$, Proceedings of the International Workshop OCRT, White Lagoon, Bulgaria, 2007.

[7] J. H. Conway and N. J. A. Sloane, A new upper bound on the minimal distance of self-dual codes, IEEE Trans. Inform. Theory 36 (1990), no. 6, 1319-1333.

[8] S. T. Dougherty, P. Gaborit, M. Harada, A. Munemasa, and P. Sole, Type IV self-dual codes over rings, IEEE Trans. Inform. Theory 45 (1999), no. 7, 2345-2360.

[9] S. T. Dougherty, P. Gaborit, M. Harada, and P. Sole, Type II codes over $\mathbb{F}_{2}+u \mathbb{F}_{2}$, IEEE Trans. Inform. Theory 45 (1999), no. 1, 32-45.

[10] S. T. Dougherty, T. A. Gulliver, and M. Harada, Extremal binary self-dual codes, IEEE Trans. Inform. Theory 43 (1997), no. 6, 2036-2047. 
[11] A. R. Hammons, P. V. Kumar, A. R. Calderbank, N. J. Sloane, and P. Sole, A linear construction for certain Kerdock and Preparata codes, Bull. Amer. Math. Soc. 29 (1993), no. 2, 218-222.

[12] M. Harada, Existence of new extremal doubly-even codes and extremal singly-even codes, Des. Codes Cryptogr. 8 (1996), no. 3, 273-283.

[13] _ The existence of a self-dual $[70,35,12]$ code and formally self-dual codes, Finite Fields Appl. 3 (1997), no. 2, 131-139.

[14] M. Harada and A. Munemasa, Some restrictions on weight enumerators of singly even self-dual codes, IEEE Trans. Inform. Theory 52 (2006), no. 3, 1266-1269.

[15] M. Harada, A. Munemasa, and V. Tonchev, A characterization of designs related to an extremal doubly-even self-dual code of length 48, Ann. Combin. 9 (2005), no. 2, 189-198.

[16] S. K. Houghten, C. W. H. Lam, L. H. Thiel, and J. A. Parker, The extended quadratic residue code is the only $(48,24,12)$ self-dual doubly-even code, IEEE Trans. Inform. Theory 49 (2003), no. 1, 53-59.

[17] W. C. Huffman, On the classification and enumeration of self-dual codes, Finite Fields Appl. 11 (2005), no. 3, 451-490.

[18] , On the decomposition of self-dual codes over $\mathbb{F}_{2}+u \mathbb{F}_{2}$ with an automorphism of odd prime order, Finite Fields Appl. 13 (2007), no. 3, 681-712.

[19] , Self-dual codes over $\mathbb{F}_{2}+u \mathbb{F}_{2}$ with an automorphism of odd order, Finite Fields Appl. 15 (2009), no. 3, 277-293.

[20] W. C. Huffman and V. Tonchev, The existence of extremal self-dual $[50,25,10]$ codes and quasisymmetric $2-(49,9,6)$, Des. Codes Cryptogr. 6 (1995), no. 2, 97-106.

$[21] \longrightarrow$, The $[52,26,10]$ binary self-dual codes with an automorphism of order 7 , in Proc. Optimal Codes and Related Topics, 127-136, Sozopol, Bulgaria, 1998.

[22] H. J. Kim, Lee-extremal self-dual codes over $\mathbb{F}_{2}+u \mathbb{F}_{2}$ of lengths 23 and 24, Finite Fields Appl. 29 (2014), 18-33.

[23] H. J. Kim, H. Lee, J. B. Lee, and Y. Lee, Construction of self-dual codes with an automorphism of order p, Adv. Math. Commun. 5 (2011), no. 1, 23-36.

[24] H. J. Kim and Y. Lee, Construction of extremal self-dual codes over $\mathbb{F}_{2}+u \mathbb{F}_{2}$ with an automorphism of odd order, Finite Fields Appl. 18 (2012), no. 5, 971-992.

[25] - Hermitian self-dual codes over $\mathbb{F}_{2^{2 m}}+u \mathbb{F}_{2^{2 m}}$, Finite Fields Appl. 25 (2014), $106-131$

[26] J.-L. Kim, New extremal self-daul codes of length 36, 38, and 58, IEEE Trans. Inform. Theory 47 (2001), no. 1, 386-393.

[27] C. L. Mallows and N. J. A. Sloane, An upper bound for self-dual codes, Inform. Control 22 (1973), 188-200.

[28] E. M. Rains, Shadow bounds for self-dual codes, IEEE Trans. Inform. Theory 44 (1998), no. $1,134-139$.

[29] H.-P. Tsai, Existence of some extremal self-dual codes, IEEE Trans. Inform. Theory 38 (1992), no. 6, 1829-1833.

[30] N. Yankov, New optimal [52, 26, 10] self-dual codes, Des. Codes Cryptogr. 69 (2013), no. 2, 151-159.

[31] N. Yankov and R. Russeva, Binary self-dual codes of lengths 52 to 60 with an automorphism of order 7 or 13, IEEE Trans. Inform. Theory 56 (2011), no. 11, 7498-7506.

[32] S. Zhang and S. Li, Some new extremal self-dual codes with lengths 42, 44, 52, and 58, Discrete Math. 238 (2001), no. 1-3, 147-150.

Institute of Mathematical Sciences

EWHa Womans University

SeOul 120-750, Korea

E-mail address: guswls41@ewha.ac.kr 